\title{
Effets du traitement des plants de riz (Oryza sativa L.) au chlorure de sodium sur la synthèse et accumulation des sucres totaux et des composés phénoliques ethano- solubles dans les feuilles et racines.
}

\author{
GOGBEU Seu Jonathan ${ }^{1 *}$, YAPO Sopie Edwige-Salomé ${ }^{1}$, GORE Bi Boh Nestor ${ }^{2}$, AYOLIE Koutoua ${ }^{1}$ \\ KOUASSI N'Dri Jacob1, NACANABO Rasmane ${ }^{1}$, DOGBO Denezon Odette ${ }^{3}$, KOUADIO Yatty Justin ${ }^{1}$ \\ 1 : Laboratoire d'Amélioration de la Production Agricole, Université Jean Lorougnon Guédé, Côte d'Ivoire \\ 2 : Laboratoire de Génétique, Université Jean Lorougnon Guédé, Côte d'Ivoire \\ 3 : Laboratoire de Biologie et Amélioration des Productions Végétales, Université Nangui Abrogoua, Cote d'Ivoire. * : \\ Auteur correspondant : e-mail : jgogbeu@yahoo.fr, contact : (+225)48626426 (Côte d'Ivoire) \\ Original submitted in on 22 nd November 2018. Published online at www.m.elewa.org/journals/ on $31^{\text {st }}$ March 2019 \\ https://dx.doi.org/10.4314/jab.v135i1.4
}

\section{RESUME}

Objectif : Ce travail a été réalisé pour évaluer la capacité du riz (Oryza sativa L.) à élaborer des stratégies d'acclimatation au stress salin en prenant la teneur des feuilles et racines en sucres totaux et composés phénoliques comme références. Ces composés sont synthétisés au cours du stress abiotique chez la plupart les plantes.

Méthodologie et Résultats : Les plants de 7,60 et 90 jours des variétés Zouhn-kinmin et Kpeulia ont été traités au $\mathrm{NaCl} 5$ ou $10 \mathrm{gL}^{-1}$, et le dosage à $0,1,2,3$ et 4 ème jours après traitement. Les résultats indiquent une quantité importante de sucres totaux avec $5 \mathrm{gL}^{-1}$ de NaCl chez les plants de Zouhn-kinmin de 60 jours [562,83 (feuilles) et 222,83 (racines) $\mu \mathrm{g}$ Glucose gMF-1]. Chez Kpeulia, l'accumulation a été maximale avec $10 \mathrm{gL}^{-1} \mathrm{de} \mathrm{NaCl}$. Chez ces variétés, la synthèse des phénols a été forte dans les feuilles et racines des plants de 90 jours avec $\mathrm{NaCl} 5 \mathrm{gL}^{-1}$.

Conclusion et Application des résultats : La teneur en sucres totaux et en composés phénoliques exprimée chez ces deux variétés montre que la variété Zouhn-kinmin serait plus tolérante au stress salin pendant la phase végétative et la variété Kpeulia à la phase de reproduction. Pour l'étude donc du comportement des plants de riz au taux souvent élevé dans le sol des engrais mal appliqués, le $\mathrm{NaCl}$ peut être utilisé.

Mots clés : Oryza sativa, stress salin, sucres totaux, composés phénoliques

\section{ABSTRACT}

Objective: This work was done to evaluate ability of rice (Oryza sativa L.) to develop salt stress acclimation strategies by taking leaf and root content of total sugars and phenolic compounds as references. These compounds are synthesized during abiotic stress in most plants

Methodology and Results: Plants of 7,60 and 90 days of Zouhn-kinmin and Kpeulia varieties were treated with $\mathrm{NaCl} 5$ or $10 \mathrm{gL}^{-1}$, and dosage at $0,1,2,3$ and 4 days after treatment. Results indicate a significant amount of total sugars with $5 \mathrm{gL}^{-1}$ of $\mathrm{NaCl}$ in Zouhn-kinmin plants of 60 days [562.83 (leaves) and 222.83 
(roots) $\mu \mathrm{g}$ Glucose gMF-1]. In Kpeulia, accumulation was maximal with $10 \mathrm{gL}^{-1}$ of $\mathrm{NaCl}$. In these varieties, phenol synthesis was strong in leaves and roots of 90-day-old plants with $\mathrm{NaCl} 5 \mathrm{gL}-1$.

Conclusion and Application of results: Total sugar and phenol compound content expressed in these two varieties shows that Zouhn-kinmin variety is more tolerant of salt stress during vegetative phase and Kpeulia variety in reproductive phase. For study of the behavior of rice plants at the often high rate in the soil of poorly applied fertilizers, $\mathrm{NaCl}$ can be used.

Keywords: Oryza sativa, salt stress, total sugars, phenolic compounds

\section{INTRODUCTION}

La salinisation des sols affecte aujourd'hui le tiers des terres agricoles et constitue un facteur limitant de la production (Drevon et al., 2001). Elle peut être naturelle ou induite par les activités agricoles comme l'irrigation des parcelles avec de l'eau de mauvaise qualité et/ou l'utilisation de certains engrais (Bartels et Nelson, 1994 ; Rubio et al., 1995). L'effet de la salinité se manifeste généralement chez les plantes par des changements morphologiques, physiologiques, biochimiques et moléculaires; ce qui affecte négativement la productivité végétale (Khan et al., 1997 ; Tafforeau, 2002 ; Ashraf et Harris, 2004). La tolérance au sel chez la plante est fonction de l'espèce, du génotype, de l'âge et de l'état physiologique des organes (El Mekkaoui, 1990). En Côte d'Ivoire, les problèmes souvent rencontrés pour la culture de riz pluvial sont liés principalement à la persistance des contraintes abiotiques dont la sécheresse, la toxicité ferreuse et la salinité des sols. La riziculture pluviale qui est la plus importante en termes de culture de riz, fournit environ $90 \%$ de la production nationale et $80 \%$ de la production de paddy (Ouattara, 2011).

\section{MATERIEL ET METHODES}

Dispositif expérimental et traitement des plants de

riz : Les plants de riz (Oryza sativa L.) âgés de 7, 60 et 90 jours ont été utilisés. Ces plants proviennent des variétés traditionnelles Kpeulia et Zouhn-kinmin) cultivées à l'Ouest de la Côte d'Ivoire. Pour l'obtention des plants, les semis ont été réalisés dans des pots en plastique contenant $10 \mathrm{~kg}$ de substrat formé de sol et de feuilles mortes des sous-bois. Les grains ont été déposés à $3 \mathrm{~cm}$ de profondeur et les pots ont été placés sous-ombrière et arrosés régulièrement avec de l'eau selon la capacité au champ. Pour chaque variété, les plants ont été répartis en 3 blocs de 30 plants selon les âges. Chaque bloc est composé de 2 sous-blocs de
Cette production demeure cependant faible eu égard à la demande en consommation. Chez les plantes de façon générale, l'acclimatation des espèces est liée à la synthèse et à l'accumulation d'un certain nombre de composés entre autres les sucres totaux et les composés phénoliques. Les sucres totaux sont des éléments carbonés primaires synthétisés et exportés dans toute la plante lors de la photosynthèse (Najdim et al., 2008). Sous stress de sécheresse et salin, les réserves amylacées sont progressivement utilisées suite à leur conversion rapide en saccharose, inhibant ainsi la synthèse de l'amidon (Geigenberger et al., 1997). Quant aux composés phénoliques, plusieurs études ont montré que leur synthèse pendant les conditions de stress participe à la résistance ou l'acclimatation des plantes (Clérivet et al., 2013). La présente étude a eu pour objectif d'évaluer la teneur des feuilles et des racines en sucres totaux et composés phénoliques chez deux variétés de riz pluvial cultivées à l'Ouest de la Côte d'Ivoire sous l'influence du chlorure de sodium.

15 plants selon la concentration de $\mathrm{NaCl}\left(5\right.$ et $\left.10 \mathrm{gL}^{-1}\right)$; soit un total de 90 plants a été utilisé pour une variété. Pour étudier l'influence du stress salin, les plants ont été traités avec une solution $\mathrm{NaCL} 5 \mathrm{gL}^{-1}$ ou $10 \mathrm{gL}^{-1}$. Aux différents âges, l'apport d'eau a été interrompu et l'arrosage a été effectué avec la solution $\mathrm{NaCl}$ selon la capacité au champ. Au jour $0,1,2,3$ et 4 après arrosage, les feuilles et les racines des plants ont été récoltées pour l'extraction et le dosage des sucres totaux et phénols.

Quantification des sucres totaux des feuilles et racines de riz: L'extraction des sucres totaux a été faite par broyage de $1 \mathrm{~g}$ de racines ou de feuilles 
fraiches dans $5 \mathrm{~mL}$ d'éthanol $80 \%(\mathrm{v} / \mathrm{v})$. Le broyat obtenu a été centrifugé à 10000 tours. min $^{-1}$ pendant 30 min à $4{ }^{\circ} \mathrm{C}$. Le surnageant a été recueilli et le culot a été repris dans $3 \mathrm{~mL}$ du solvant et traité dans les mêmes conditions précédentes. Les deux surnageants additionnés ont constitué l'extrait et servi au dosage des sucres totaux. Le dosage des sucres totaux a été fait selon la méthode de Dubois et al. (1956). Une quantité de $0,2 \mathrm{~mL}$ de phénol $5 \%(\mathrm{v} / \mathrm{v})$ a été ajouté à $0,2 \mathrm{~mL}$ d'extrait. Ce mélange a été complété à $1 \mathrm{~mL}$ avec de l'eau distillée auquel on a ajouté $1 \mathrm{~mL}$ d'acide sulfurique concentré $96 \%(\mathrm{v} / \mathrm{v})$. Après une incubation de 5 min dans un bain à $100{ }^{\circ} \mathrm{C}$, le milieu réactionnel a été refroidi à l'obscurité pendant $30 \mathrm{~min}$. La quantité de sucres totaux a été évaluée au spectrophotomètre à $480 \mathrm{~nm}$ contre un témoin ne contenant pas d'extrait. Elle est estimée à l'aide d'une courbe étalon réalisée avec une solution de glucose (200 $\left.\mathrm{gmmL}^{-1}\right)$ et exprimée en microgramme de glucose par gramme de matière fraiche ( $\mu$ g Glucose gMF-1).

Estimation des composés phénoliques éthanosolubles des feuilles et racines du riz: L'extraction des composés phénoliques a été faite selon la méthode de Gogbeu et al. (2012). Une masse de $5 \mathrm{~g}$ de feuilles ou de racines fraiches a été broyé en présence de $5 \mathrm{~mL}$ d'éthanol $80 \%(\mathrm{v} / \mathrm{v})$. Le broyat obtenu a été centrifugé à 3000 tours. min $^{-1}$ pendant $12 \mathrm{~min}$. Après

\section{RESULTATS}

Comparaison de la teneur initiale des sucres totaux et des composés phénoliques dans les feuilles et racines des variétés zouhn-kinmin et kpeulia : La teneur en sucres totaux des organes a été plus importante dans les feuilles que les racines excepté les plants de 90 jours de la variété zouhn-kinmin (Tableau 1). Toutefois, les feuilles de kpeulia ont été plus riches en sucres totaux que celles de zouhn-kinmin. Cette teneur a augmenté avec l'âge des plants. De 214,50 $\mu \mathrm{g}$ Glucose gMF-1 $^{-1}$ chez les plants de 7 jours, elle a atteint 460,17 $\mu$ g Glucose gMF-1 au bout de 90 jours de semis. Dans les racines par contre, ce sont les plants de zouhn-kinmin qui ont synthétisé le plus de sucres totaux. Chez ce dernier, la teneur a diminué avec l'âge des plants. Initialement de 175,33 $\mu \mathrm{g}$ Glucose gMF-1 dans les plants de 7 jours, elle a diminué pour atteindre centrifugation, le surnageant a été récupéré et ajusté à $10 \mathrm{~mL}$ avec de l'éthanol $80 \%(\mathrm{v} / \mathrm{v})$. Cet extrait a été utilisé pour le dosage des composés phénoliques éthano-solubles. Le dosage a été fait selon la méthode de Singleton en utilisant le réactif de Folin-Ciocalteu (Ragaee et al., 2012). Pour ce faire, $0,8 \mathrm{~mL}$ de bicarbonate de sodium $\left(\mathrm{Na}_{2} \mathrm{CO}_{3}\right) 7,5 \%(\mathrm{~m} / \mathrm{v})$ a été ajouté à $0,2 \mathrm{~mL}$ d'extrait phénolique. Après $5 \mathrm{~min}$ d'incubation à $25^{\circ} \mathrm{C}, 1 \mathrm{~mL}$ du réactif de Folin-Ciocalteu $0,5 \mathrm{~N}$ a été ajouté. Le mélange réactionnel a été homogénéisé puis incubé pendant $30 \mathrm{~min}$ à $28^{\circ} \mathrm{C}$ à l'obscurité. La lecture de l'absorbance a été faite au spectrophotomètre à $765 \mathrm{~nm}$ contre un témoin ne contenant pas d'extrait phénolique. La quantité de phénols contenus dans l'extrait phénolique a été estimée à l'aide d'une courbe d'étalonnage réalisé avec l'acide gallique (100 $\left.\mathrm{mgL}^{-1}\right)$ et exprimée en milligramme équivalent d'acide gallique par gramme de matière fraîche (mg AG gMF-1).

Analyse statistique des données: Les données recueillies ont été soumises à une analyse de la variance (ANOVA) à un ou deux critères de classification en utilisant le logiciel SPSS 11.5 au seuil de $5 \%$. Lorsque $\mathrm{P} \leq 5 \%$, la différence est dite significative. Les groupes homogènes sont alors déterminés par la méthode de Duncan.

la valeur de 70,66 $\mu \mathrm{g}$ Glucose gMF-1 $^{-1}$ au 90ème jour après semis, soit une diminution de plus de $100 \mu \mathrm{g}$ Glucose gMF-1 $^{-1}$. Quant à la teneur en composés phénoliques des feuilles, elle a évolué de la même manière chez les deux variétés (Tableau I). Au fur et à mesure que l'âge des plants a augmenté, la quantité de phénols a augmenté également. Les feuilles des plants de 7 jours de la variété zouhn-kinmin ont été plus riches

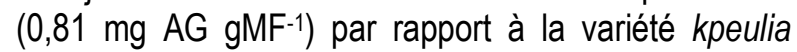
$\left(0,46 \mathrm{mg} \mathrm{AG} \mathrm{gMF}^{-1}\right)$. Mais, lorsque l'âge a augmenté, ce sont les plants de la variété kpeulia qui ont synthétisé et accumulé le plus de phénols. Par contre dans les racines, chez la variété zouhn-kinmin, la teneur a augmenté jusqu'au 60ème jour avant de

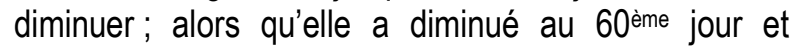
augmenté après chez la variété kpeulia. 
Tableau 1 : Evolution de la teneur en sucres totaux ( $\mu \mathrm{g}$ Glucose $\mathrm{gMF}^{-1}$ ) et composés phénoliques (mg AG gMF-1) dans les feuilles et racines des plants de riz de différents âges

\begin{tabular}{|c|c|c|c|c|}
\hline \multirow{2}{*}{$\begin{array}{c}\text { Composés } \\
\text { biochimiques }\end{array}$} & \multirow[t]{2}{*}{ Organes } & \multirow{2}{*}{$\begin{array}{c}\text { Âges des } \\
\text { organes (jour) }\end{array}$} & \multicolumn{2}{|c|}{ Variété de riz } \\
\hline & & & Zouhn-kinmin & kpeulia \\
\hline \multirow[t]{2}{*}{ Sucres totaux } & Feuilles & $\begin{array}{l}7 \\
60 \\
90 \\
\end{array}$ & $\begin{array}{r}198,66 \pm 01,66 b \\
232,00 \pm 16,66 c \\
43,66 \pm 00,83 a \\
\end{array}$ & $\begin{array}{l}214,50 \pm 00,83 a \\
418,67 \pm 00,00 b \\
460,17 \pm 18,17 b\end{array}$ \\
\hline & Racines & $\begin{array}{l}7 \\
60 \\
90 \\
\end{array}$ & $\begin{array}{r}175,33 \pm 08,33 c \\
104,83 \pm 10,83 b \\
70,66 \pm 00,00 a\end{array}$ & $\begin{array}{l}50,30 \pm 05,00 b \\
76,50 \pm 09,16 c \\
55,33 \pm 15,00 a\end{array}$ \\
\hline \multirow{2}{*}{$\begin{array}{l}\text { Composes } \\
\text { phénoliques }\end{array}$} & Feuilles & $\begin{array}{l}7 \\
60 \\
90\end{array}$ & $\begin{array}{l}0,81 \pm 0,40 a \\
1,53 \pm 0,17 b \\
1,75 \pm 0,02 b\end{array}$ & $\begin{array}{l}0,46 \pm 0,02 a \\
2,04 \pm 0,50 b \\
2,53 \pm 0,09 b\end{array}$ \\
\hline & Racines & $\begin{array}{l}7 \\
60 \\
90\end{array}$ & $\begin{array}{l}0,71 \pm 0,02 a \\
2,37 \pm 0,17 b \\
0,49 \pm 0,02 a\end{array}$ & $\begin{array}{l}1,26 \pm 0,61 c \\
0,56 \pm 0,09 a \\
0,95 \pm 0,61 b\end{array}$ \\
\hline
\end{tabular}

Pour chaque organe et composé biochimique, les valeurs suivies de la même lettre alphabétique dans une colonne ne sont pas statistiquement différentes au seuil de $5 \%$ selon le test de Duncan

Effet du traitement de la variété Zouhn-kinmin au $\mathrm{NaCl}$ sur la synthèse des sucres totaux dans les feuilles et racines : La teneur en sucres totaux chez la variété Zouhn-kinmin a varié avec l'âge des plants, le type d'organes et la durée du traitement. Dans les plants témoins, la teneur en sucres totaux a été importante dans les feuilles et racines des plants de 7 et 60 jours (Tableau 2). Toutefois, les plus fortes valeurs ont été enregistrées chez les plants de 60 jours pour les feuilles et, les plants de 7 jours pour les racines. Lorsque les plants ont été traités au $\mathrm{NaCl}$, la synthèse et l'accumulation des sucres totaux ont également varié en fonction des organes et du temps de traitement. Dans les feuilles et racines des plants traités au $\mathrm{NaCl} 5 \mathrm{gL}^{-1}$, l'accumulation maximale a été enregistrée au 4ème jour [les feuilles des plants âgés de 7 jours (260,33 $\mu \mathrm{g}$ Glucose gMF-1) et 60 jours $(562,83$ $\mu \mathrm{g}$ Glucose $\left.\left.\mathrm{gMF}^{-1}\right)\right]$, au $2^{\mathrm{ème}}$ et $4^{\mathrm{ème}}$ jours pour les racines des plants de $7(104,50 \mu \mathrm{g}$ Glucose gMF-1) et 60 jours $(222,83 \mu \mathrm{g}$ Glucose gMF-1). Quant aux plants âgés de 90 jours, les valeurs maximales ont été

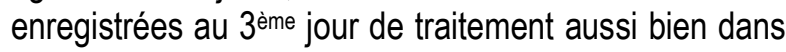

les feuilles que dans les racines. Avec $10 \mathrm{gL}^{-1}$ de $\mathrm{NaCl}$, pour les plants âgés de 7 jours, la teneur des sucres totaux a diminué durant l'expérimentation dans les racines par rapport aux témoins. Par contre, dans les feuilles au 3ème jour, de la valeur initiale de $198 \mu \mathrm{g}$ Glucose gMF-1, elle est passée à $354 \mu \mathrm{g}$ Glucose gMF1. Quant aux plants de 60 et 90 jours, dans les feuilles, les valeurs maximales ont été enregistrées respectivement au 1er (462 $\mu \mathrm{g}$ Glucose gMF-1) et au

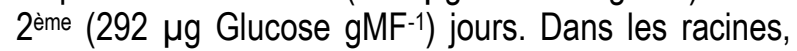
c'est au 4 ème (les plants âgés de 60 jours) et au 1er (les plants âgés de 90 jours) jours que l'accumulation des sucres totaux a été maximale. D'une manière générale, c'est dans les feuilles que la synthèse et l'accumulation des sucres totaux ont augmenté avec le temps de traitement. Toutefois, la forte synthèse des sucres totaux a été enregistrée dans les feuilles et racines des plants traités au $\mathrm{NaCl} 5 \mathrm{gL}^{-1}$ par rapport à ceux traités au $\mathrm{NaCl} 10 \mathrm{gL}^{-1}$. Ces valeurs se retrouvent chez les plants de 60 jours [feuilles $\left(562,83 \mu \mathrm{g}\right.$ Glucose gMF-1) $^{-1}$ et racines $\left(222,83 \mu \mathrm{g}\right.$ Glucose $\left.\left.\mathrm{gMF}^{-1}\right)\right]$. 
Tableau 2 : Effet de chlorure de sodium sur la synthèse et l'accumulation des sucres totaux ( $\mu$ g Glucose gMF-1) dans les feuilles et les racines des plants de riz de la variété Zouhn-kinmin

\begin{tabular}{|c|c|c|c|c|c|c|c|}
\hline \multirow[t]{2}{*}{ Organes } & \multirow{2}{*}{$\begin{array}{l}\mathrm{NaCl} \\
\left(\mathrm{gL}^{-1}\right)\end{array}$} & \multirow{2}{*}{$\begin{array}{l}\text { Ages } \\
\text { (jours) }\end{array}$} & \multicolumn{5}{|c|}{ Temps après application de $\mathrm{NaCl}$ (jours) } \\
\hline & & & témoin & 1 & 2 & 3 & 4 \\
\hline \multirow{6}{*}{ Feuilles } & \multirow{3}{*}{5} & 7 & $198,66 \pm 01,66 b$ & $238,66 \pm 18,33 b$ & $241,16 \pm 09,16 b$ & $259,50 \pm 27,50 b$ & $260,33 \pm 35,00 b$ \\
\hline & & 60 & $232,00 \pm 16,66 c$ & $522,00 \pm 23,33 c$ & $348,66 \pm 11,66 c$ & $468,66 \pm 05,00 c$ & $562,83 \pm 22,50 c$ \\
\hline & & 90 & $43,66 \pm 00,83 a$ & $81,16 \pm 07,50 a$ & $119,50 \pm 10,83 a$ & $179,50 \pm 00,83 a$ & $158,66 \pm 06,66 a$ \\
\hline & \multirow{3}{*}{10} & 7 & $198,66 \pm 01,66 \mathrm{~b}$ & $246,16 \pm 14,16 b$ & $274,50 \pm 14,16 a$ & $354,50 \pm 07,50 \mathrm{c}$ & $341,16 \pm 14,16 c$ \\
\hline & & 60 & $232,00 \pm 16,66 c$ & $462 \pm 60,00 c$ & $382,83 \pm 04,16 c$ & $332,00 \pm 16,66 b$ & $257,83 \pm 99,16 a$ \\
\hline & & 90 & $43,66 \pm 00,83 a$ & $189,50 \pm 12,50 a$ & $292,83 \pm 14,16 b$ & $261,16 \pm 14,16 a$ & $262,00 \pm 15,00 b$ \\
\hline \multirow{6}{*}{ Racines } & \multirow{3}{*}{5} & 7 & $175,33 \pm 08,33 c$ & $75,33 \pm 05,00 a$ & $104,50 \pm 25,83 b$ & $81,16 \pm 34,16 a$ & $58,66 \pm 05,00 b$ \\
\hline & & 60 & $104,83 \pm 10,83 b$ & $92,00 \pm 03,33 b$ & $132,00 \pm 16,66 c$ & $176,16 \pm 04,16 c$ & $222,83 \pm 00,83 c$ \\
\hline & & 90 & $70,66 \pm 00,00 a$ & $101,16 \pm 14,16 c$ & $48,66 \pm 05,00 a$ & $142,83 \pm 45,83 b$ & $38,66 \pm 01,66 a$ \\
\hline & \multirow{3}{*}{10} & 7 & $175,33 \pm 08,33 c$ & $122,00 \pm 38,33 b$ & $81,16 \pm 00,83 a$ & $121,16 \pm 34,16 b$ & $111,16 \pm 04,16 a$ \\
\hline & & 60 & $104,83 \pm 10,83 b$ & $97,00 \pm 23,33 a$ & $114,50 \pm 04,16 c$ & $123,66 \pm 28,83 b$ & $133,66 \pm 03,33 c$ \\
\hline & & 90 & $70,66 \pm 00,00 a$ & $142,83 \pm 05,83 c$ & $102,00 \pm 25,00 b$ & $84,50 \pm 00,83 a$ & $126,16 \pm 24,16 b$ \\
\hline
\end{tabular}

Pour chaque organe et concentration $\mathrm{NaCl}$, les valeurs suivies de la même lettre alphabétique dans une colonne ne sont pas statistiquement différentes au seuil de $5 \%$ selon le test de Duncan 
Teneur en sucres totaux des feuilles et racines de la variété kpeulia traitée au $\mathrm{NaCl}$ : Chez la variété Kpeulia, la quantité de sucres totaux a varié avec l'âge et le type d'organes ainsi que la durée du traitement des plants de riz au NaCl (Tableau 3). Dans les feuilles des plants témoins, les plus grandes valeurs ont été enregistrées chez les plants âgés de 90 jours, suivi des plants de 60 jours. Par contre dans les racines, l'accumulation a été plus importante chez les plants de 60 jours suivis de ceux de 90 jours. Lorsque ces différents plants ont été traités au $\mathrm{NaCl}$, la synthèse et l'accumulation des sucres totaux ont varié selon l'âge et la durée de traitement. Dans les feuilles et les racines après traitement au $\mathrm{NaCl} 5 \mathrm{gL}^{-1}$, l'accumulation maximale de sucres totaux a été enregistrée respectivement au 1er (feuilles des plants âgés de 60 jours) et 4ème jours (les feuilles des plants âgés 7 jours)

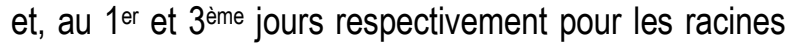
des plants âgés de 60 et 7 jours. Quant aux plants âgés de 90 jours, les valeurs maximales ont été enregistrées

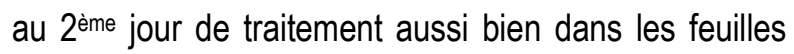
que dans les racines. Lorsque les plants ont été traités au $\mathrm{NaCl} 10 \mathrm{gL}^{-1}$, pour les plants âgés de 7 jours, une légère accumulation des sucres totaux a été observée

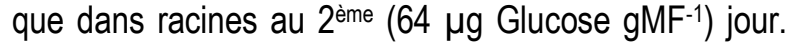
Quant aux plants âgés de 60 et 90 jours, l'accumulation des sucres totaux dans les feuilles a été maximale respectivement au $4{ }^{\mathrm{ème}}$ ( $588 \mu \mathrm{g}$ Glucose gMF-1 $\left.^{-1}\right)$ et $2^{\text {ème }}$ (1704 $\mu \mathrm{g}$ Glucose gMF-1) jours. Dans les racines, c'est au 2 ème jour que l'accumulation des sucres totaux a été maximale avec 167 (plants âgés de 60 jours) et 159 (plants âgés de 90 jours) $\mu$ g Glucose gMF-1. Chez la variété Kpeulia, la synthèse des sucres totaux a été forte dans les feuilles et racines des plants ayant subi un traitement au $\mathrm{NaCl} 10 \mathrm{gL}^{-1}$ par rapport à ceux ayant subi un traitement au $\mathrm{NaCl} 5 \mathrm{gL}^{-1}$. Au niveau des quantités de sucres totaux synthétisés dans les feuilles et racines quel que soit la concentration de $\mathrm{NaCl}$ utilisée, le temps d'échantillonnage et l'âge du matériel végétal, la différence a été significative $(p \leq 0,05)$. 
Tableau 3 : Effet de chlorure de sodium sur la synthèse et l'accumulation des sucres totaux ( $\mu$ g Glucose gMF-1) dans les feuilles et les racines des plants de riz de la variété kpeulia

\begin{tabular}{|c|c|c|c|c|c|c|c|}
\hline \multirow[t]{2}{*}{ Organes } & \multirow{2}{*}{$\begin{array}{l}\mathrm{NaCl} \\
\left(\mathrm{gL}^{-1}\right)\end{array}$} & \multirow{2}{*}{$\begin{array}{l}\text { Ages } \\
\text { (jours) }\end{array}$} & \multicolumn{5}{|c|}{ Temps après application de $\mathrm{NaCl}$ (jours) } \\
\hline & & & témoin & 1 & 2 & 3 & 4 \\
\hline \multirow{6}{*}{ Feuilles } & \multirow{3}{*}{5} & 7 & $214,50 \pm 00,83 a$ & $59,50 \pm 07,50 a$ & $95,33 \pm 00,00 a$ & $222,00 \pm 11,66 a$ & $228,67 \pm 63,33 a$ \\
\hline & & 60 & $418,67 \pm 00,00 b$ & $542,00 \pm 23,33 b$ & $377,00 \pm 21,66 b$ & $353,67 \pm 53,33 b$ & $297,83 \pm 12,50 c$ \\
\hline & & 90 & $460,17 \pm 18,17 b$ & $447,00 \pm 10,33 c$ & $528,68 \pm 61,66 c$ & $349,50 \pm 09,17 b$ & $307,00 \pm 78,33 b$ \\
\hline & \multirow{3}{*}{10} & 7 & $214,50 \pm 00,83 a$ & $99,50 \pm 00,83 a$ & $140,33 \pm 21,61 a$ & $99,50 \pm 02,50 a$ & $98,66 \pm 11,66 a$ \\
\hline & & 60 & $418,67 \pm 00,00 b$ & $505,33 \pm 61,66 b$ & $436,17 \pm 02,50 \mathrm{~b}$ & $363,67 \pm 26,66 b$ & $587,83 \pm 25,83 b$ \\
\hline & & 90 & $460,17 \pm 18,17 b$ & $775,33 \pm 88,33 c$ & $1704,50 \pm 185,33 c$ & $1535,33 \pm 288,33 c$ & $462,00 \pm 76,66 c$ \\
\hline \multirow{6}{*}{ Racines } & \multirow{3}{*}{5} & 7 & $50,30 \pm 05,00 b$ & $52,00 \pm 05,00 a$ & $55,33 \pm 01,66 b$ & $113,66 \pm 06,66 c$ & $60,33 \pm 05,00 b$ \\
\hline & & 60 & $76,50 \pm 09,16 c$ & $122,83 \pm 19,16 c$ & $72,00 \pm 11,66 b$ & $72,83 \pm 00,83 a$ & $42,00 \pm 01,66 a$ \\
\hline & & 90 & $55,33 \pm 15,00 a$ & $76,16 \pm 02,50 b$ & $122,83 \pm 27,50 \mathrm{c}$ & $84,50 \pm 12,50 b$ & $81,16 \pm 27,50 c$ \\
\hline & \multirow{3}{*}{10} & 7 & $50,30 \pm 05,00 a$ & $48,66 \pm 08,33 a$ & $63,66 \pm 01,66 a$ & $40,33 \pm 00,00 a$ & $37,00 \pm 01,66 a$ \\
\hline & & 60 & $76,50 \pm 09,16 b$ & $97,00 \pm 01,66 b$ & $167,00 \pm 01,66 b$ & $97,83 \pm 15,83 b$ & $95,33 \pm 13,33 c$ \\
\hline & & 90 & $55,33 \pm 15,00 c$ & $133,66 \pm 16,66 c$ & $159,50 \pm 07,50 b$ & $103,67 \pm 05,00 b$ & $76,16 \pm 04,16 b$ \\
\hline
\end{tabular}

Pour chaque organe et concentration $\mathrm{NaCl}$, les valeurs suivies de la même lettre alphabétique dans une colonne ne sont pas statistiquement différentes au seuil de $5 \%$ selon le test de Duncan 
Synthèse et l'accumulation des composés phénoliques éthano-solubles dans les feuilles et racines des plants de Zouhn-kinmin traités au $\mathrm{NaCl}$ : La teneur des composés phénoliques a varié avec l'âge des organes des plants de riz (Tableau 4). Dans les feuilles, les plus grandes valeurs ont été enregistrées chez les plants âgés de 60 et 90 jours. Par contre dans les racines, ce sont les plants âgés de 60 jours qui ont enregistré une quantité importante de composés phénoliques. Lorsque les plants ont été traités au $\mathrm{NaCl}$, la synthèse et l'accumulation des composés phénoliques ont varié en fonction des organes et du temps d'échantillonnage. Ainsi, après traitement des plants au $\mathrm{NaCl} 5 \mathrm{gL}^{-1}$, dans les feuilles et racines des plants âgés de 7 jours, l'accumulation des composés phénoliques a été maximale

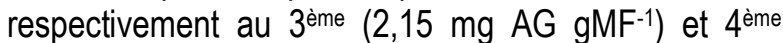
$\left(0,38 \mathrm{mg} \mathrm{AG} \mathrm{gMF}^{-1}\right)$ jours. Toutefois, cette teneur dans les racines a été inférieure à celle du témoin. Quant aux plants âgés de 60 et 90 jours, l'accumulation des composés phénoliques a été maximale dans les feuilles au 4 ème $(2,61)$ et $2^{\text {ème }}(2,38)$ jours. Dans les racines, cette concentration a entrainé une baisse

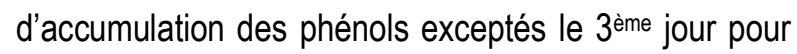
les plants âgés de 90 jours. Avec $10 \mathrm{gL}^{-1} \mathrm{de} \mathrm{NaCl}$, dans les plants âgés de 7 jours, l'accumulation des composés phénoliques a baissé dans les racines par rapport au témoin avec une faible accumulation au 4ème (0,49 mg AG gMF-1) jour. Par contre dans les feuilles, d'une valeur initiale de $0,80 \mathrm{mg}$ AG gMF-1, $^{-1}$ l'accumulation des composés phénoliques a été maximale au 3ème jour avec 1,12 mg AG gMF-1. Quant aux plants âgés de 60 et 90 jours, l'accumulation des composés phénoliques a été maximale dans les feuilles au 1er (2,44 mg AG gMF-1) et 3ème (2,69 mg AG gMF-1) jours et dans les racines au 1 er $\left(1,32 \mathrm{mg} \mathrm{AG} \mathrm{gMF}^{-1}\right)$ jour pour les plants de 90 jours. Cette teneur est restée inférieur à celle du témoin pour les racines des plants âgés de 60 jours. En effets, les fortes teneurs en composés phénoliques ont été obtenues qu'au niveau des feuilles et racines des plants âgés de 90 jours traités avec $5 \mathrm{gL}^{-1}$ de $\mathrm{NaCl}$. L'analyse statistique effectuée a montré que la teneur en composés phénoliques au cours de l'expérimentation a varié considération en fonction des temps d'échantillonnage $(p<0,05)$. 
Gogbeu et al., J. Appl. Biosci. 2019 Effets du traitement des plants de riz au chlorure de sodium sur la synthèse et accumulation des sucres totaux et des composés phénoliques ethano-solubles dans les feuilles et racines.

Tableau4 : Effet de chlorure de sodium sur la synthèse et l'accumulation des composés phénoliques (mg AG gMF-1) dans les feuilles et les racines des plants de riz de la variété Zouhn-kinmin

\begin{tabular}{|c|c|c|c|c|c|c|c|}
\hline \multirow{2}{*}{ Organes } & \multirow{2}{*}{$\begin{array}{l}\mathrm{NaCl} \\
\left(\mathrm{gL}^{-1}\right)\end{array}$} & \multirow{2}{*}{ Ages (jours) } & \multicolumn{5}{|c|}{ Temps après application de $\mathrm{NaCl}$ (jours) } \\
\hline & & & témoin & 1 & 2 & 3 & 4 \\
\hline \multirow[t]{2}{*}{ Feuilles } & 5 & $\begin{array}{c}7 \\
60 \\
90\end{array}$ & $\begin{array}{l}0,81 \pm 0,40 a \\
1,53 \pm 0,17 b \\
1,75 \pm 0,02 b\end{array}$ & $\begin{array}{l}1,76 \pm 0,07 b \\
1,43 \pm 0,39 a \\
1,81 \pm 0,83 b\end{array}$ & $\begin{array}{l}0,95 \pm 0,11 a \\
1,21 \pm 0,06 a \\
2,38 \pm 0,03 b\end{array}$ & $\begin{array}{l}2,15 \pm 0,09 a \\
2,55 \pm 1,58 a \\
2,90 \pm 0,03 a\end{array}$ & $\begin{array}{l}1,74 \pm 0,07 a \\
2,61 \pm 0,80 b \\
1,79 \pm 0,73 a\end{array}$ \\
\hline & 10 & $\begin{array}{c}7 \\
60 \\
90 \\
\end{array}$ & $\begin{array}{l}0,81 \pm 0,40 \mathrm{a} \\
1,53 \pm 0,17 \mathrm{~b} \\
1,75 \pm 0,02 \mathrm{~b}\end{array}$ & $\begin{array}{l}1,03 \pm 0,01 a \\
2,44 \pm 1,01 b \\
1,13 \pm 0,27 a\end{array}$ & $\begin{array}{l}1,02 \pm 0,04 a \\
2,02 \pm 0,50 b \\
1,37 \pm 0,63 a\end{array}$ & $\begin{array}{l}1,12 \pm 0,03 a \\
1,41 \pm 0,12 a \\
2,69 \pm 0,25 b\end{array}$ & $\begin{array}{l}0,82 \pm 0,03 a \\
1,12 \pm 0,17 b \\
1,79 \pm 0,03 c\end{array}$ \\
\hline \multirow[t]{2}{*}{ Racines } & 5 & $\begin{array}{c}7 \\
60 \\
90\end{array}$ & $\begin{array}{l}0,71 \pm 0,02 b \\
2,37 \pm 0,17 c \\
0,49 \pm 0,02 a\end{array}$ & $\begin{array}{l}0,10 \pm 0,07 a \\
1,32 \pm 0,01 b \\
0,72 \pm 0,18 c\end{array}$ & $\begin{array}{l}0,11 \pm 0,30 a \\
0,73 \pm 0,28 b \\
1,06 \pm 0,33 c\end{array}$ & $\begin{array}{l}0,31 \pm 0,09 a \\
1,41 \pm 0,07 b \\
1,55 \pm 0,02 b\end{array}$ & $\begin{array}{l}0,38 \pm 0,06 a \\
1,96 \pm 0,24 b \\
0,54 \pm 0,02 a\end{array}$ \\
\hline & 10 & $\begin{array}{c}7 \\
60 \\
90\end{array}$ & $\begin{array}{l}0,71 \pm 0,02 b \\
2,37 \pm 0,17 c \\
0,49 \pm 0,02 a\end{array}$ & $\begin{array}{l}0,33 \pm 0,10 a \\
0,90 \pm 0,06 b \\
1,32 \pm 0,36 c\end{array}$ & $\begin{array}{l}0,11 \pm 0,02 a \\
1,51 \pm 0,36 c \\
0,92 \pm 0,36 b\end{array}$ & $\begin{array}{l}0,28 \pm 0,01 a \\
1,25 \pm 0,05 b \\
0,53 \pm 0,12 a\end{array}$ & $\begin{array}{l}0,49 \pm 0,06 a \\
1,54 \pm 0,03 b \\
0,59 \pm 0,07 a\end{array}$ \\
\hline
\end{tabular}

Pour chaque organe et concentration $\mathrm{NaCl}$, les valeurs suivies de la même lettre alphabétique dans une colonne ne sont pas statistiquement différentes au seuil de $5 \%$ selon le test de Duncan 
Effets du traitement des plants de la variété Kpeulia au $\mathrm{NaCl}$ sur la synthèse des composés phénoliques dans les feuilles et racines : L'analyse du tableau 5 montre que la teneur des organes en composés phénoliques a varié avec le temps de traitement et l'âge des organes des plants de riz. Dans les feuilles des plants témoins, les plus grandes valeurs ont été enregistrées dans les plants âgés de 90 jours, suivi des plants âgés de 60 jours. Par contre, dans les racines, ce sont les plants âgés de 7 et 90 jours qui ont enregistré une quantité importante de composés phénoliques. Lorsque ces plants ont été traités au $\mathrm{NaCl}$, la synthèse et l'accumulation des composés phénoliques ont varié également en fonction des organes et du temps d'échantillonnage. Après traitement au $\mathrm{NaCl} 5 \mathrm{gL}^{-1}$, dans les feuilles et racines des plants âgés de 7 jours, l'accumulation maximale a été enregistrée respectivement au $3^{\text {ème }}(1,75 \mathrm{mg} \mathrm{AG}$
$\left.\mathrm{gMF}^{-1}\right)$ et 1 er $\left(1,44 \mathrm{mg} \mathrm{AG} \mathrm{gMF}^{-1}\right)$ jours. Quant aux plants âgés de 60 et 90 jours, dans les feuilles, l'accumulation maximale des composés phénoliques a été enregistrée respectivement au 1 er $(4,13 \mathrm{mg} \mathrm{AG}$ gMF-1 $^{-1}$ et 4 ème (3,75 mg AG gMF-1) jours. Concernant les racines, l'accumulation a été maximale au 2 ème jour. Avec $10 \mathrm{gL}^{-1} \mathrm{de} \mathrm{NaCl}$, pour les plants âgés de 7 jours, l'accumulation des composés phénoliques dans les feuilles et les racines a été maximale respectivement

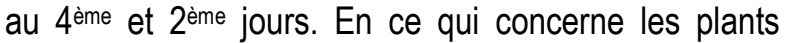
âgés de 60 et 90 jours, les feuilles ont enregistré une accumulation maximale de composés phénoliques respectivement au 4ème $\left(2,50 \mathrm{mg} \mathrm{AG} \mathrm{gMF}^{-1}\right)$ et 1er (3,08 mg AG gMF-1) jours. Par contre dans les racines, la

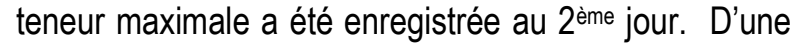
manière générale, les teneurs les plus élevées ont été obtenues dans les feuilles et racines des plants âgés de 90 jours avec les traitements $5 \mathrm{gL}^{-1} \mathrm{de} \mathrm{NaCl}$. 
Gogbeu et al., J. Appl. Biosci. 2019 Effets du traitement des plants de riz au chlorure de sodium sur la synthèse et accumulation des sucres totaux et des composés phénoliques ethano-solubles dans les feuilles et racines.

Tableau 5 : Effet de chlorure de sodium sur la synthèse et l'accumulation des composés phénoliques (mg AG gMF-1) dans les feuilles et les racines des plants de riz de la variété kpeulia

\begin{tabular}{|c|c|c|c|c|c|c|c|}
\hline \multirow[t]{2}{*}{ Organes } & \multirow{2}{*}{$\begin{array}{l}\mathrm{NaCl} \\
\left(\mathrm{gL}^{-1}\right)\end{array}$} & \multirow[t]{2}{*}{ Ages (jours) } & \multicolumn{5}{|c|}{ Temps après application de $\mathrm{NaCl}$ (jours) } \\
\hline & & & témoin & 1 & 2 & 3 & 4 \\
\hline \multirow{6}{*}{ Feuilles } & & 7 & $0,46 \pm 0,02 a$ & $0,54 \pm 0,04 a$ & $0,75 \pm 0,16 a$ & $1,75 \pm 0,50 a$ & $1,51 \pm 0,72 a$ \\
\hline & 5 & 60 & $2,04 \pm 0,50 b$ & $4,13 \pm 0,47 c$ & $2,41 \pm 0,24 b$ & $2,23 \pm 0,81 b$ & $2,13 \pm 0,19 b$ \\
\hline & & 90 & $2,53 \pm 0,09 b$ & $3,45 \pm 0,72 b$ & $2,93 \pm 0,27 \mathrm{c}$ & $3,22 \pm 0,21 c$ & $3,75 \pm 0,09 c$ \\
\hline & & 7 & $0,46 \pm 0,02 a$ & $1,11 \pm 0,21 a$ & $1,03 \pm 0,61 a$ & $0,71 \pm 0,03 a$ & $1,29 \pm 0,35 a$ \\
\hline & 10 & 60 & $2,04 \pm 0,50 \mathrm{~b}$ & $1,88 \pm 0,14 b$ & $2,11 \pm 0,77 b$ & $1,33 \pm 0,00 b$ & $2,50 \pm 0,88 b$ \\
\hline & & 90 & $2,53 \pm 0,09 b$ & $3,08 \pm 0,37 c$ & $2,91 \pm 0,15 c$ & $2,76 \pm 0,39 c$ & $2,88 \pm 0,84 b$ \\
\hline \multirow{6}{*}{ Racines } & & 7 & $1,26 \pm 0,61 \mathrm{c}$ & $1,44 \pm 0,29 b$ & $0,69 \pm 0,14 a$ & $0,80 \pm 0,14 a$ & $0,71 \pm 0,21 \mathrm{c}$ \\
\hline & 5 & 60 & $0,56 \pm 0,09 a$ & $0,87 \pm 0,41 a$ & $1,62 \pm 0,70 b$ & $0,49 \pm 0,02 b$ & $0,30 \pm 0,01 a$ \\
\hline & & 90 & $0,95 \pm 0,61 b$ & $0,97 \pm 0,04 a$ & $1,58 \pm 0,15 b$ & $0,76 \pm 0,00 \mathrm{a}$ & $1,32 \pm 0,41 b$ \\
\hline & & 7 & $1,26 \pm 0,61 \mathrm{c}$ & $0,38 \pm 0,15 a$ & $2,16 \pm 0,45 c$ & $1,41 \pm 0,28 b$ & $0,66 \pm 0,07 a$ \\
\hline & 10 & 60 & $0,56 \pm 0,09 a$ & $0,88 \pm 0,15 b$ & $1,02 \pm 0,35 a$ & $0,99 \pm 0,11 a$ & $0,32 \pm 0,01 b$ \\
\hline & & 90 & $0,95 \pm 0,61 b$ & $0,72 \pm 0,13 b$ & $1,67 \pm 0,33 b$ & $1,00 \pm 0,08 a$ & $1,52 \pm 0,12 c$ \\
\hline
\end{tabular}

Pour chaque organe et concentration $\mathrm{NaCl}$, les valeurs suivies de la même lettre alphabétique dans une colonne ne sont pas statistiquement différentes au seuil de $5 \%$ selon le test de Duncan 


\section{DISCUSSION}

Le traitement des plants avec des molécules élicitrices induit l'activation d'une série de réactions métaboliques. Celles-ci conduisent à la mise en place des mécanismes d'acclimatation qui sont entre autres, la synthèse et l'accumulation de composés solubles. En effet, dans les mécanismes d'acclimatation des plantes, la compartimentation vacuolaire ou l'exclusion des ions toxiques ont été largement évoqués par plusieurs auteurs (Blumwald et al., 2004 ; Munns et Tester, 2008). Pour ces auteurs, le surplus du sodium dans le cytoplasme est rejeté vers l'apoplasme ou encore au cloisonnement des ions $\mathrm{Na}^{+}$et $\mathrm{Cl}^{-}$au niveau cellulaire et intracellulaire pour éviter leurs concentrations élevées dans le cytoplasme en particulier dans les cellules de mésophile. Ce mécanisme contribue ainsi au maintien de la croissance cellulaire en conditions de stress salin (Sairam et Tyagi, 2004). Chez le riz (Oryza sativa L), le traitement des plants au chlorure de sodium $(\mathrm{NaCl})$ a conduit à la synthèse et à l'accumulation des sucres totaux et des composés phénoliques dans les feuilles et les racines. Dans les plants témoins, la teneur en sucres totaux a été importante chez la variété Kpeulia. En fonction des organes, après traitement des plants, la teneur des sucres totaux a varié selon le temps d'échantillonnage. Les fortes valeurs ont été enregistrées plus dans les feuilles que dans les racines. Ces valeurs se sont retrouvées chez les plants de 60 jours. La présence des sucres totaux dans les feuilles à une quantité importante par rapport aux racines est surement liée à l'intensité photosynthétique dans cette partie de la plante. En effet, les feuilles des plants contiennent plus de chlorophylles, donc les produits de la photosynthèse seraient plus élevés à cet endroit. Or, les sucres totaux font partir des produits de la photosynthèse. De même, Sebane (2015) a observé dans son étude sur le pourpier de mer (Atriplex halimus) une forte accumulation des sucres totaux dans les feuilles que dans les racines en condition de stress salin. L'augmentation de la synthèse et de l'accumulation des sucres totaux dans ces organes laissent croire qu'ils jouent un rôle dans l'acclimatation du riz au stress salin. En effet, plusieurs auteurs ont montré que les sucres totaux renforçaient le contenu du cytoplasme, ce qui augmenterait la pression osmotique de la cellule par rapport au milieu extérieur (El Midaoui et al., 2007). Ce phénomène permet à la plante de réaliser l'osmose facilement. La baisse de la quantité des sucres totaux à un moment de la vie de la plante montre que les sucres totaux sont mobilisés comme substrats dans des voies métaboliques. D'une manière générale, c'est à $5 \mathrm{gL}^{-1}$ de $\mathrm{NaCl}$ qu'une augmentation importante de la quantité des sucres totaux a été obtenue. Pour des auteurs Hassan et al. (2008) qui ont travaillé sur l'orge (Hordeum vulgare L.) et Regueragui (2005) sur la tomate (Solanum lycopersicum L.), la teneur en sucres augmente avec l'augmentation de la concentration du sel dans le milieu. C'est ce qui explique cette teneur légèrement importante par rapport aux plants témoins. Mais à $10 \mathrm{gL}^{-1}$, hormis les plants de riz de 90 jours de la variété Kpeulia cette concentration a créé un effet inhibiteur sur la synthèse des sucres totaux chez le riz. La teneur des sucres totaux a varié cependant selon les variétés et les organes de riz. Ces mêmes observations ont été évoquées par Ottow et al. (2005) et Zerrad et al. (2006). Dans cette étude, les plants de 60 jours seraient à leur activité végétative maximale, ce qui pourrait expliquer la quantité de sucres totaux plus importante à ce niveau. En ce qui concerne la quantité de composés phénoliques dans les feuilles et les racines, elle a été plus importante chez la variété Kpeulia que la variété Zouhn-kinmin. Lorsque le stress a été appliqué par le traitement des plants au $\mathrm{NaCl}$, la quantité des composés phénoliques a également augmenté. Les composés phénoliques participent à la rigidification cellulaire par le renforcement des parois cellulaire (Majourhat et Baaziz, 2004). Certains de ces composés sont sollicités dans d'autres voies de biosynthèse. La chute de la quantité des composés phénoliques à un moment donné de l'expérimentation montre que d'autres protéines enzymatiques utilisent ces composés. En effet, les travaux réalisés par Gogbeu et al, (2012) sur le manioc ont montré que les polyphénoloxydases utilisaient les composés phénoliques comme substrat. De même, les études réalisées par Dogbo et al, (2008) chez la même plante ont montré que les teneurs des composés phénoliques variaient avec le temps après élicitation à l'acide salicylique. Les peroxydases et les polyphénoloxydases seraient surement à la base de la diminution de cette quantité (Hiraga et al., 2001; Dogbo et al., 2008). Selon Grant et Lamb (2006), la capacité d'une espèce végétale à résister aux microorganismes est souvent corrélée avec la teneur en composés phénoliques. Cette quantité importante de phénols dans les organes de riz contribuerait donc à l'acclimatation de la plante au stress salin. Au vue de la quantité de sucres totaux et de phénols synthétisés, la variété Zouhn-kinmin serait plus tolérante au stress salin du stade 7 jours jusqu'àu 60 ème jour de la vie 


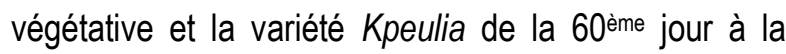

\section{CONCLUSION}

La réponse des variétés Zouhn-kinmin et Kpeulia au stress salin a montré que ces plants de riz synthétisent des sucres totaux et des composés phénoliques dans les feuilles et racines. Cependant, ces effets varient en fonction de l'intensité du stress et de la variété. La variété Zouhn-kinmin s'est montrée tolérante au stress

\section{REFERENCES BIBLIOGRAPHIQUES}

Ashraf M. and Harris., 2004. Potential biochemical indicators of salinity tolerance in plants. Plant Science., 166: 3-6.

Bartels D. and Nelson D., 1994. Approaches to improve stress tolerance using molecular genetics. Plant Cellular Environment, 17: 659- 667.

Blumwald E., Grover A. and Good A.G., 2004. Breeding for abiotic stress resistance: challenges and opportunities. "New directions for a diverse planet ॥. In: Proceedings of the $4^{\text {th }}$ International Crop Science Congress, 26 September-1 October, Brisbane, Australia. www.cropscience. org.au.

Dogbo D.O., Békro-Mamyrbekova J.A., Békro Y-A., Sié R.S., Gogbeu S.J. et Traoré A. (2008). Influence de l'acide salicylique sur la synthèse de la phénylalanine ammonia-lyase, des polyphénoloxydases et l'accumulation des composes phénoliques chez le manioc (Manihot esculenta Crantz). Science \& Nature, 5(1): 1-13.

Drevon J.J., Saadallah K., Hajji M. and Abdelly C., 2001. Genotypic variability for tolerance to salinity of N2-fixing common bean (Phaseolus vulgaris L.), Agronomy, 21: 675-682.

Dubois M., Gilles K., Hamilton J., Rebers P. and Smith F., 1956. Colorimetric method for determination of sugars and related substances. Analytical Chemistry, 28 (3): 350356.

El Mekkaoui M., 1990. Etude des mécanismes de tolérance à la salinité chez le blé dur ( $T$. durum Desf.) et l'orge ( $H$. vulgare L.) : Recherche de tests précoces de sélection. Thèse en Science Agronomique., Université de Nice Sophia, Montpellier, 191p

El Midaoui M., Benbella M., Aït Houssa A., Ibriz M.et Talouizte A., 2007. Contribution à l'étude de quelques mécanismes d'adaptation à la phase de reproduction (90ème jour).

salin à la phase végétative, alors que la variété Kpeulia tolère le sel à la phase de reproduction. Pour l'étude du comportement des plants de riz au taux souvent élevé dans le sol des engrais mal appliqués, le $\mathrm{NaCl}$ peut être utilisé pour mimer.

salinité chez le tournesol cultivé (Helianthus annus L.). Revue HTE, 136: 29-34.

Geigenberger P., Reimholz R., Geiger M., Merlo L., Canale V. and Stitt M., 1997. Resolution of sucrose and starch metabolism in potato tubers in response to short-term water deficit. Planta, 201: 502 -518.

Gogbeu S.J., Dogbo D.O., Zohouri G.P., N'Zué B. and Bekro-Mamyrbekova J.A., 2012. Induction of polyphenoloxidases activities and phenolic compounds accumulation in cells and plants elicited of cassava (Manihot esculenta Cranz). Journal of Scientific Research and Reviews, 1(1): 7-14.

Hassan I., DellaL A., Belkhodja M.et Kaid-Harche M., 2008. Effet de la salinité sur l'eau et certains osmolytes chez l'orge (Hordeum Vulgare). European Journal of Scientific Research, 23 (1): 61-69.

Hiraga S., Sasaki K., Ito H., Ohashi Y. and Matsui H., 2001. A Large Family of Class III Plant Peroxidases, Plant Cell Physiology, 42(5): 462-468.

Khan M.A., Hamid A., Salahuddin A. B.M., Quasem A. and Karim M.A., 1997. Effect of sodium chloride on growth, photosynthesis and mineral ions accumulation of different types of rice (Oryza sativa). Journal Agronomy and Science, 149- 161.

Majourhat K. et Baaziz M., 2004. Diversité et caractéristiques des peroxydases foliaires du palmier dattier. Congrès international de biochimie, Marrakech, Maroc, 153-156.

Munns R. and Tester M., 2008. Mechanisms of salinity tolerance. Annual Review of Plant Biology, 59: 651- 681.

Munns R. and Termaat A., 1986. Whole-plant responses to salinity. Australian Journal of plant Physiology, 13(1): 143- 160. 
Najdim B., El jaafari S., jlibène M. et Jacquemin J.M., 2008. Application des marqueurs moléculaires dans l'amélioration du blé tendre pour la résistance aux maladies et aux insectes. Biotechnologie Agronomie, Société et Environnement, 1 : 17- 35.

Ottow E., Brinker M., Fritz E., Teichmann T., Kaiser W., Brosche M., Kangasjarvi J., Jiang X. and Polle A., 2005. Populus euphratica Displays Apoplastic Sodium Accumulation, Osmotic Adjustment by Decreases in Calcium and Soluble Carbohydrates, and Develops Leaf Succulence under Salt Stress. Plant Physiology, 139. 1762-1772.

Ouattara Z.F., 2011. Analyse de la compétitivité du riz local en Côte d'Ivoire. Rapport du PRESAO, (3), 8p.

Ragaee S., Abdel-Hal E.S.M. and Noaman K., 2012. Antioxydant activity and nutrient composition of selected cereals for food use. Food Chemistry, $98: 32-38$.

Regueragui A., 2005. Contribution à l'étude de l'influence de la salinité sur le couple tomateVerticillium : Conséquences physiologiques et impact sur la bio protection des tomates contre la verticilliose. Thèse de doctorat. Université Mohammed V. Agdal. Rabat, Maroc, 100-103.

Rubio F., Gassmann W. and Schroeder J.L., 1995. Sodium driven potassium uptake by the plant potassium transporter HKT1 and mutations conferring salt tolerance. Science, 270: 16601663.

Sairam R.K. and Tyagi A., 2004. Physiology and molecular biology of salinity stress tolerance in plants. Curriculum of Science, 86: 407- 421.

Sebane R.F., 2015. Action combine de la salinité et de l'acide salicylique sur les réponses biochimiques de deux espèces: Atriplex halimus L. et Atriplex canescens (Pursh) Nutt. Master Science de la Vie et de la Terre, Université d'ORAN, ALGERIE, $49 \mathrm{p}$.

Tafforeau M., 2002. Etude des phases précoces de la transduction des signaux environnementaux chez le lin : une approche protéomique. Thèse de doctorat en Biochimie végétale. Université de Rouen. France. 255p.

Zerrad W., Hillali S., Mataoui B.S., Elantri E. et Elhmyene A., 2006. Etude des mécanismes biochimiques et moléculaires de résistance au stress hydrique de deux variétés de blé dur. Lebanese Science Journal, $9: 27-36$ 